\title{
La evidencia científica en la información al consumidor: las declaraciones nutricionales y de propiedades saludables (health claims) en los alimentos
}

\section{Scientific evidence in consumer information: nutrition and health claims on food}

\author{
ANDREU PALOU OLIVER ${ }^{1,2}$, MARIONA PALOU MARCH ${ }^{3}$ \\ 'Laboratori de Biologia Molecular, Nutrició i Biotecnologia (LBNB). Universitat de les Illes Balears \\ (UIB); ${ }^{2}$ Centro de Investigación Biomédica en Red sobre Obesidad y Nutrición (CIBERobn); \\ ${ }^{3}$ Alimentómica S.L. Campus de la UIB Palma de Mallorca. e-mail: andreu.palou@uib.es
} Fecha de recepción: 12/11/2015 · Fecha de aceptación: 03/05/2016

\section{RESUMEN}

El Reglamento Europeo 1924/2006 sobre declaraciones de salud (nutricionales y de propiedades saludables o health claims) gobierna el uso de estas declaraciones de forma armonizada en toda la Unión Europea. Representa el resultado y es, a su vez, el principal elemento generador del cambio cualitativo más importante en el sector de la alimentación de las últimas 4 décadas, el que conecta al consumidor con el binomio alimentación-salud. Desde incluso antes de su implementación a principios de 2007 , se ha situado en la base de los principales criterios y tendencias que dirigen los nuevos desarrollos en la industria agroalimentaria. En el fondo está la preocupación por los principales problemas de salud de las sociedades desarrolladas, la obesidad, diabetes, enfermedades cardiovasculares y otras enfermedades crónicas de nuestro tiempo que tienen que ver, fundamentalmente con la alimentación y el sedentarismo, con un enorme coste en términos de salud y económicos. En 2006 los estados miembros de la UE recogieron y presentaron a la Comisión Europea para su evaluación por EFSA unos 44 mil eslóganes de salud en los alimentos existentes en el mercado comunitario, muchos de ellos repetitivos - obviamente descartables y quedaron en 4637; actualmente sólo hay unos 260 health claims autorizados. Hoy las propiedades saludables de los alimentos deben haber sido demostradas científicamente mediante estudios del más alto nivel de calidad, incluyendo la demostración de los efectos en humanos. Deben reevaluarse algunas de las previsiones que no ha sido posible materializar tras 10 años de desarrollo de esta legislación, periodo en el que todos (legisladores, empresas, consumidores, administración y científicos) hemos ido aprendiendo en la aplicación y descifrado de una normativa que los expertos consideran como una de la más comple- 


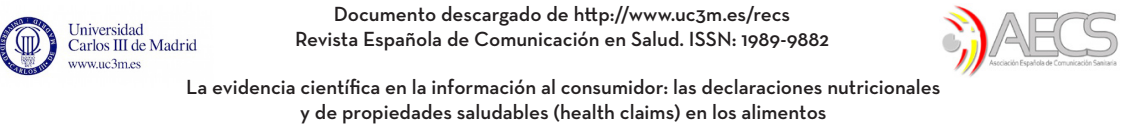

jas del ámbito Europeo. Problemas pendientes mayúsculos son: a) los "perfiles nutricionales": ¿cualesquiera alimentos, incluso los más energéticos, pueden llevar health claims?); y b) la evaluación de los extractos de plantas y otros productos 'botánicos' que componen muchos de los complementos alimenticios, un subsector que también demanda armonización a nivel Europeo.

Palabras clave: declaraciones de propiedades saludables; propiedades nutricionales; alimentos funcionales; Reglamento (CE) 1924/2006.

\section{ABSTRACT}

Regulation (EC) No 1924/2006 on nutrition and health claims made on foods ("the Regulation") was adopted in 2006 to govern the use of health claims in the labelling, presentation and advertising of foods in the European Union. It represents the result and is, in turn, the principal generating element of the most important qualitative change in the food sector of last 4 decades. The Regulation connects the consumer with the binomial foodhealth. It conforms the basis for the main criteria and tendencies directing strategic developments in the agro-food sector. The main health problems in the developed societies are those related with obesity, diabetes, cardiovascular diseases and other chronic disorders, closely related with food and sedentary life with an enormous cost in terms of health and economy. In 2006 about 44000 slogans were gathered by the EU member states from their markets and presented to the European Commission for evaluation by EFSA. After filtering repetitions and obvious cases, 4636 were set for evaluation. Currently only 260 health claims are authorized. The change is that nowadays to allow a health claim its effects have to be demonstrated (substantiated by robust high quality scientific studies), including the demonstration of the effects in humans. All stakeholders (legislators, companies, consumers, administration and scientists) have been learning during these 9 years of the implementation and developments of the Regulation, which is considered one of the more complex piece of legislation ever adopted in the EU. Pending issues are: a) nutritional profiles: any food, even the most energetics, should be allowed to bear health claims?); and b) the current rules concerning health claims on botanicals. Key words: health claims; nutritional properties; functional foods; Regulation (EC) 1924/2006. 


\section{INTRODUCCIÓN}

Con la progresiva implementación y desarrollo en los últimos 9 años del Reglamento Europeo CE) no 1924/2006 ,relativo a las declaraciones nutricionales y de propiedades saludables (health claims) en los alimentos (EU, 2007), Europa se ha situado en la vanguardia del mundo desarrollado, en cuanto a las garantías que hoy aseguran al consumidor que los mensajes que recibe sobre propiedades de los alimentos relacionadas con la salud son veraces; en el justo sentido de que tales beneficios se ajustan a una evidencia científica sólida. Sin embargo, quedan todavía algunos aspectos por desarrollar, como la definición de los perfiles nutricionales a los que debe ajustarse un alimentos que pretenda, voluntariamente, llevar claims; así como la posible reconsideración de la aplicabilidad a los extractos botánicos de los mismos criterios aplicados hasta ahora para evaluar la evidencia científica. También, se está generalizando un cierto abuso de la reformulación de alimentos, por el procedimiento de añadirles nutrientes esenciales $\mathrm{u}$ otros bioactivos que, por tener acreditado un claim, pueden así extender el mensaje de salud al alimento reformulado: en muchos de estos casos, aunque se mantenga el principio de veracidad, puede ser que lo que se traslada al consumidor aún se aleje demasiado de "toda la verdad".

La condición esencial para que un health claim sea autorizado es que lo que afirme esté bien demostrado por la evidencia científica, mediante estudios y pruebas reconocidas ampliamente por la comunidad científica, según una evaluación muy rigurosa, del máximo nivel de exigencia, realizada por la EFSA (European Food Safety Authority, Autoridad Europea en
Seguridad Alimentaria). La segunda condición es que el claim sea fácilmente entendible por el consumidor, lo que no es un tema menor, si bien la conjugación se ambos aspectos se escapa del presente resumen.

Se entiende por «declaración de propiedades saludables» (health claim) cualquier mensaje o representación que no sea obligatorio con arreglo a la legislación comunitaria o nacional, incluida cualquier forma de representación pictórica, gráfica o simbólica, que afirme, sugiera o dé a entender que existe una relación entre una categoría de alimentos, un alimento o uno de sus constituyentes, y la salud (EU, 2007).

TIPOS DE DECLARACIONES DE SALUD: NUTRICIONALES Y DE PROPIEDADES SALUDABLES

En Europa son posibles dos tipos generales de declaraciones de salud en los alimentos: las "nutricionales" (declaraciones sobre la composición) y las de "propiedades saludables" (traducción de health claims que aparece en la transposición de la legislación europea a la española) que se refieren a los efectos de mejora o mantenimiento de funciones $\mathrm{u}$ otros aspectos relativos a la salud o saludables. Existe, de acuerdo con lo que dispone el Reglamento, un "registro de la UE" de las declaraciones que han sido autorizadas, y también de las rechazadas, que se actualiza periódicamente y que contiene información sobre la lista de claims nutricionales y sus condiciones de uso; están tanto los health claims autorizados (sus condiciones de uso y sus restricciones, en su caso) como los no autorizados (y las razones de su desestimación), así como a otras disposiciones legales y, en su caso, 
las disposiciones particulares de los países miembros:[http://www.aecosan.msssi. gob.es/AECOSAN/web/seguridad_alimentaria/subdetalle/registro_comunitario.shtml]

\section{DECLARACIONES NUTRICIONALES}

Se refieren a lo que contienen los alimentos, se elimina la confusión de términos como 'light', contiene [nombre del nutriente u otra sustancia]; ', 'enriquecido', 'sin calorías', etc., dado que se han establecido límites o referencias cuantitativas para las declaraciones aprobadas, de forma que la ambigüedad ya no es posible en este tipo de declaraciones. Se entiende por «declaración nutricional» cualquier declaración que afirme, sugiera o dé a entender que un alimento posee propiedades nutricionales benéficas específicas con motivo del aporte energético (valor calórico) que proporciona, que proporciona en un grado reducido o incrementado, o que no proporciona; y/o de los nutrientes $u$ otras sustancias que contiene, que contiene en proporciones reducidas o incrementadas, o bien que no contiene (artículo 2.4 del Reglamento) (EU, 2007). Solamente se autorizan las declaraciones nutricionales si están incluidas en el Anexo (actualizado) del Reglamento y si se ajustan a las condiciones fijadas en el mismo. Las modificaciones del Anexo se adoptan por el Comité Permanente de la Cadena Alimentaria, previa consulta a la EFSA. Así, en la actualidad tenemos 30 tipos de declaraciones nutricionales aceptadas, a saber: bajo valor energético; valor energético reducido; sin aporte energético; bajo contenido de grasa; sin grasa; bajo contenido de grasas saturadas; sin grasas saturadas; bajo contenido de azúcares ; sin azúcares; sin azúcares añadidos, bajo contenido de sodio/sal; muy bajo contenido de sodio/sal; sin sodio o sin sal; sin sodio o sin sal añadida; fuente de fibra; alto contenido de fibra; fuente de proteínas; alto contenido de proteínas; fuente de [nombre de las vitaminas] y/o [nombre de los minerales] ; alto contenido de [nombre de las vitaminas] y/o [nombre de los minerales]; contiene [nombre del nutriente u otra sustancia]; mayor contenido de [nombre del nutriente]; contenido reducido de [nombre del nutriente]; light/lite (ligero) ; naturalmente/natural; fuente de ácidos grasos omega-3; alto contenido de ácidos grasos omega-3; alto contenido de grasa monoinsaturada; alto contenido de grasa poliinsaturada; alto contenido de grasa insaturada.

Las condiciones de uso de cada una de ellas pueden ser consultadas en el propio registro: http://ec.europa.eu/food/safety/ labelling_nutrition/claims/nutrition_claims/ index_en.htm. Se trata de un anexo abierto en el sentido de que, en función de los nuevos conocimientos se pueden añadir (validadas por EFSA) nuevas declaraciones o modificar las existentes.

\section{DECLARACIONES DE PROPIEDADES SALUDABLES}

Se consideran 3 tipos de declaraciones de declaraciones de propiedades saludables (health claims):

1. Las denominadas "Funcionales" o del Artículo 13 del Reglamento, que son: a) las relativas al crecimiento, desarrollo $y$ funciones del organismo; b) las que se refieren a funciones psicológicas y comportamentales; y c) las referidas a adelgazar o al control del peso corporal

2. Las declaraciones de "reducción del riesgo de enfermedad" o del Artículo 
14.1(a) del Reglamento, que son cualquier declaración de propiedades saludables que afirme, sugiera o dé a entender que el consumo de una categoría de alimentos, un alimento o uno de sus constituyentes reduce significativamente un factor de riesgo de aparición de una enfermedad humana.

3. Las declaraciones que se refieran al "desarrollo y la salud de los niños" o del Artículo 14.1(b).

En la actualidad hay registradas unas 260 declaraciones de propiedades saludables, que son las que se pueden utilizar, la mayoría de ellas referentes a efectos de vitaminas y minerales esenciales, cuyos beneficios (esencialmente para prevenir efectos adversos de dietas deficientes o carenciales) eran ya bien conocidos desde hace años. Menos de una tercera parte corresponden a efectos beneficiosos demostrados en años más recientes, tales como las de los ácidos grasos esenciales (omega 3 y omega 6), ácidos grasos monoinsaturados, insaturados y poliinsaturados, los polifenoles del aceite de oliva, los esteroles y estanoles vegetales, extractos de tomate; algunos tipos de fibra, nueces, ciertas bebidas conteniendo carbohidratos y electrolitos, azúcares-alcoholes sustitutos de azúcares, yogurt con menos lactosa, y ciertos preparados para usos nutricionales particulares.

Una idea del gran filtrado que se ha producido para llegar a la situación actual de sólo 260 claims autorizados en el mercado Europeo es que, en 2006 se recogieron más de 44.000 eslóganes por los diferentes estados miembros de la UE. En 2007, después de eliminar repeticiones y descartar casos obvios, quedaron 4637 para su evaluación. De ellas, la mayoría de las evaluaciones por el Panel de la EFSA fue- ron desfavorables y los claims asociados han sido rechazados; otra gran parte (más de 1500 solicitudes) han quedado pendientes de evaluación por corresponder a unos 500 claims propuestos para plantas o preparaciones/extractos de plantas (se les suele denominar 'botánicos'), cuya consideración legal, para algunos productos o como categoría, no está armonizada en el conjunto Europeo: un mismo producto puede ser considerado un complementos alimenticio en un país y como un producto medicinal en otro país. Esta falta de armonización genera agravios comparativos y mucha controversia pues, en este momento, las exigencias (en cuanto a declaraciones de propiedades saludables) pueden ser mayores para los complemento (a los que se les exige una demostración, generalmente en base a estudios de intervención en humanos) mientras una extensa tradición de uso seguro (30 años) de un medicamento le permite comunicar beneficios sin necesidad de aportar estudios preclínicos o clínicos, siempre que no se considere un producto peligroso en las condiciones de uso que tenga atribuidas. En 2010 la Comisión Europea dictaminó que estos productos botánicos se excluyeran (temporalmente) de la evaluación por la EFSA. De este modo, el proceso de evaluación ha quedado fragmentado en 2 procesos: a) el que se aplica de modo general; y b) un procedimiento (por definir) que, en su momento (cuando se alcance una decisión) se aplicará a los productos botánicos. Creemos que la decisión dependerá de que pueda avanzarse (con desarrollos paralelos en los Estados) en la legislación conjunta sobre complementos alimenticios (de momento existente sólo para nutrientes esenciales) y que en el futuro deberá incluir otros bioactivos y po- 
sibles preparados o extractos de plantas (botánicos).

\section{DECLARACIONES CON PROTECCIÓN DE DATOS EN PROPIEDAD}

A título de ejemplo, podemos citar 2 declaraciones de este tipo, actualmente vigentes, una referente a la fibra de remolacha azucarera (contribuye a que aumente el volumen de las heces) y otra referente a los hidratos de carbono: contribuyen a la recuperación de la función muscular normal (contracción) después de un ejercicio físico de gran intensidad o de larga duración que conduce a la fatiga muscular y al agotamiento del glucógeno almacenado en los músculos esqueléticos. Pero ha habido otras que ya no pueden estar vigentes, por el hecho de que la protección es por un periodo 5 años desde su autorización, a partir de los cuales la declaración ya puede utilizarse por otros operadores económicos.

Se trata de declaraciones que han sido acreditadas con "datos en propiedad"; es decir que los datos científicos u otro tipo de información de la solicitud, necesarios para la autorización, no pueden utilizarse en beneficio de un solicitante posterior durante un período de 5 años. Esta protección (con ciertas analogías con las patentes) se otorga sólo en el caso de que no se hubiera podido autorizar la declaración de propiedades saludables (el Panel de EFSA emite un juicio al respecto en el trámite de la evaluación) si el solicitante anterior no hubiera aportado los datos protegidos por derechos de propiedad industrial. Este aspecto es de indudable importancia por lo que supone de fuerte incentivo a la I+D+i en el sector de la alimentación, por el valor añadido que alcanzan los productos que consiguen ser acreditados con health claims específicos y con derechos de propiedad: las empresas que invierten en estudios científicos para intentar demostrar propiedades saludables de los alimentos, pueden ver así recompensada su inversión.

\section{DECLARACIONES DE "REDUCCIÓN DEL RIESGO DE ENFERMEDAD”.}

Estas declaraciones no pueden expresar directamente una reducción del riesgo de contraer la enfermedad sino (la diferencia es sutil) la reducción significativa de un factor de riesgo de aparición de la enfermedad humana a la que se refiera.

En la actualidad hay registradas 9 declaraciones de este tipo, a favor de los siguientes nutrientes o productos:

Calcio y vitamina D: reducen la pérdida de mineral de los huesos en mujeres postmenopáusicas. Esta pérdida es un factor de riesgo para las fracturas óseas osteoporóticas.

Ácidos grasos monoinsaturados y/o poliinsaturados: la sustitución de grasas saturadas con insaturadas reduce el colesterol sanguíneo. El colesterol elevado es un factor de riesgo para la enfermedad cardíaca coronaria.

Vitamina D: ayuda a reducir el riesgo de caídas asociadas a inestabilidad postural y debilidad muscular. Estas caídas son un factor de riesgo de fracturas óseas en hombres y mujeres a partir de los 60 años de edad.

Betaglucanos de cebada: reducen el colesterol. El colesterol elevado es un factor de riesgo para la enfermedad cardíaca coronaria.

Betaglucanos de avena; reducen el colesterol. El colesterol elevado es un factor de riesgo para la enfermedad cardíaca coronaria. 
Chicles sin azúcar: ayudan a reducir los ácidos de la placa. Los ácidos de la placa son un factor de riesgo para el desarrollo de caries

Chicles sin azúcar: ayudan a reducir la desmineralización dental. La desmineralización dental es un factor de riesgo para el desarrollo de caries.

Esteroles vegetales y ésteres de estanoles vegetales. Reducen el colesterol sanguíneo. El colesterol elevado es un factor de riesgo para la enfermedad cardíaca coronaria.

Esteroles vegetales (extraídos de plantas, libres o esterificados con ácidos grasos de calidad alimentaria). Reducen el colesterol sanguíneo. El colesterol elevado es un factor de riesgo para la enfermedad cardíaca coronaria. O bien. "Se ha demostrado que los fitosteroles (esteroles vegetales) disminuyen/reducen la colesterolemia. Una tasa elevada de colesterol constituye un factor de riesgo en el desarrollo de cardiopatías coronarias".

Además, junto a cada declaración están las condiciones de su utilización; por ejemplo, en este último caso son: «Debe informarse al consumidor de que el efecto beneficioso se obtiene con una ingesta diaria de 1,5 a $3 \mathrm{~g}$ de fitoesteroles (esteroles vegetales). Solo podrá hacerse referencia a la magnitud del efecto para los alimentos incluidos en las siguientes categorías: grasas amarillas para untar, productos lácteos, mayonesa y aliños para ensaladas. Cuando se haga referencia a la magnitud del efecto, deberá comunicarse al consumidor la variación "del $7 \%$ al 10 \%" para los alimentos que aporten una ingesta diaria de 1,5 a 2,4 g de fitoesteroles o la variación "del $10 \%$ al 12,5\%" para los alimentos que aporten una ingesta diaria de 2,5 a $3 \mathrm{~g}$ de fitoesteroles, así como el período a partir del cual se obtiene el efecto: "de dos a tres semanas"".

En el caso de las declaraciones de reducción del riesgo de enfermedad, el etiquetado o, de no existir éste, la presentación - la publicidad, deberá incluir asimismo una exposición en la que se indique que la enfermedad a la que se refiere la declaración posee múltiples factores de riesgo y que la alteración de uno de estos factores de riesgo puede tener o no un efecto benéfico.

\section{DECLARACIONES SALUDABLES RELA- TIVAS AL "DESARROLLO Y SALUD DE LOS NIÑOS"}

En Enero de 2016 se habían registrado 11 declaraciones relativas al desarrollo y salud de los niños. Sin extendernos en detalles, notemos que el término 'niños' resulta algo impreciso en relación a muchos aspectos de la legislación alimentaria (más allá de lo legislado en la UE para niños de 0 a 3 años de edad, y los términos aprobados para diversos nutrientes incluidos en las listas positivas de las correspondientes disposiciones legales específicas); y tampoco está claro (debe analizarse caso por caso) que numerosas declaraciones de propiedades saludables funcionales, autorizadas para la población general, no puedan aplicarse a niños de otras edades, o a adolescentes. En todo caso, las 11 expresamente autorizadas, saludables, en niños son:

Ácido $\alpha$-linolénico y ácido linoleico, ácidos grasos esenciales: Los ácidos grasos esenciales son necesarios para el crecimiento $y$ el desarrollo normales de los niños.

Calcio y vitamina D: El calcio y la vitamina $D$ son necesarios para el crecimiento y el desarrollo normales de los huesos en los niños. 
Calcio: El calcio es necesario para el crecimiento y el desarrollo normales de los huesos en los niños.

Vitamina $D$ : La vitamina $D$ es necesaria para el crecimiento y el desarrollo normales de los huesos en los niños.

Proteínas: Las proteínas son necesarias para el crecimiento y el desarrollo normales de los huesos en los niños.

Fósforo: El fósforo es necesario para el crecimiento y el desarrollo normales de los huesos en los niños.

Yodo: El yodo contribuye al crecimiento normal de los niños.

Hierro: El hierro contribuye al desarrollo cognitivo normal de los niños

Ácido docosahexaenoico (DHA): La ingesta de ácido docosahexaenoico (DHA) contribuye al desarrollo visual normal de los niños hasta los 12 meses de edad.

Como ejemplos de las condiciones de uso, las correspondientes al hierro (son análogas para otras vitaminas y minerales esenciales) son: "Esta declaración sólo puede utilizarse en relación con alimentos que son, como mínimo, fuente de hierro de acuerdo con la declaración fuente de [nombre de las vitaminas] y/o [nombre de los minerales] que figura en el anexo del Reglamento (CE) no 1924/ 2006", lo cual supone (por $100 \mathrm{~g} \circ \mathrm{ml}$ ) una ingesta del $15 \%$ de la cantidad diaria recomendada.

Para el caso del DHA, las condiciones de uso son: "Se informará al consumidor de que el efecto beneficioso se obtiene con una ingesta diaria de $100 \mathrm{mg}$ de DHA. Cuando la declaración se haga con respecto a preparados de continuación, el alimento debe contener al menos un $0,3 \%$ del total de ácidos grasos como DHA".

\section{DECLARACIONES DE PROPIEDADES} SALUDABLES "FUNCIONALES"

Son las más numerosas, se encuentran registradas 230 declaraciones de este tipo, dirigidas a la población general. Esta lista se forma con las "funcionales genéricas" que son las que, se autorizaron después de haberse considerado/evaluado todas las existentes en el mercado (según lo propuesto por los estados miembros de la UE) (Artículos 13.1 y 13.5 del Reglamento), junto con unas pocas basadas en nueva ciencia (tramitadas y evaluadas de acuerdo con el artículo 13.5 del Reglamento).

Las declaraciones funcionales deben acreditar un efecto beneficioso (en el ámbito de la salud) ya sea por contar con evidencia directa de la mejora de una determinada función o proceso beneficioso o por contar con evidencia de efecto sobre un biomarcador apropiado, en el sentido de que el efecto sobre el biomarcador es considerado beneficioso o posiblemente beneficioso.

Las declaraciones de propiedades saludables deben siempre ir acompañarse de una serie de mensajes sobre aspectos generales, importantes en una buena alimentación y nutrición, tales como: a) la importancia de una dieta variada y equilibrada y un estilo de vida saludable; b) la cantidad de alimento y el patrón de consumo requeridos para obtener el efecto benéfico declarado; c) en su caso, una declaración dirigida a las personas que deberían evitar el consumo del alimento; $y$ una advertencia adecuada en relación con los productos que pueden suponer un riesgo para la salud si se consumen en exceso.

\section{LA SUBSTANCIACIÓN CIENTÍFICA}

Sería prolijo extendernos en los detalles de esta evaluación pero debe enfatizar- 
se que el mandato de que "las declaraciones de propiedades saludables en los alimentos y las nutricionales deben estar substanciadas científicamente" es lo esencial del nuevo Reglamento. Aunque la incertidumbre a lo largo del proceso ha sido importante, poco a poco se han ido consolidando criterios, especialmente a partir de la propia experiencia de evaluación a cargo del Panel científico NDA de la EFSA. Esta experiencia se ha traducido, en primer lugar, en las guías para la preparación de los dosieres y de toda la información recomendable para documentar una declaración de propiedades saludables. Esencialmente se mantienen los criterios establecidos por el Panel en 2007 (EFSA Panel On Dietetic Products $\mathrm{NaAN}, 2007)$, en su mayor parte reproducidos en la versión más recientemente actualizada ((EFSA Panel On Dietetic Products $\mathrm{NaAN}, 2016$ ), pero cuya comparación permite aquilatar algunas nuevas tendencias; en particular (aunque controvertida), la posible ampliación de lo que podemos entender por población sana. No debe olvidarse que la frontera entre alimentos y medicamentos viene determinada por la forma de expresión del claim (en un alimento no debe referirse a "prevención tratamiento o curación de enfermedades"), y por la población diana (si el producto se destina a pacientes, entonces se entiende como "tratamiento" y ello corresponde a un medicamento). Un aspecto clave en el desarrollo y oportunidades en el marco de esta nueva legislación europea sobre declaraciones de propiedades saludables y nutricionales, es que la substanciación científica se apoya en el uso de factores de riesgo y/o biomarcadores medibles, reproducibles y validables. Se trata de parámetros
- conjuntos de los mismos, que pueden servir de base para la demostración (en estudios apropiados y en humanos) de eventuales relaciones causa-efecto entre el consumo de un alimento (o componentes del mismo) y un efecto (beneficioso) para la salud. Sin embargo, la realidad es que se carece de biomarcadores para muchas funciones, incluyendo algunas que se presentan como muy interesantes (efectos antiinflamatorios, estrés metabólico, efecto antioxidante) que no son aceptables si no son específicos sobre un proceso (cuyo cambio es beneficioso) y, por otra parte, el número de factores de riesgo de enfermedad aceptados por la EFSA es más bien escaso. De este modo, la falta de este tipo de biomarcadores (biomarcadores de salud, nuevos factores de riesgo) supone actualmente una de las principales limitaciones para la expansión del sector de la alimentación en el campo de la salud (véase BIOCLAIMS, 2010).

\section{PERSPECTIVA}

Es cierto que desde la época anterior a 2007 , cuando la casi exclusiva preocupación era la seguridad, ya se trabajaba en la regulación de los futuros health claims (Palou, A.; Pico, C. \& Bonet, M.L., 2004), y que se ha avanzado mucho. Se ha pretendido, quizás por primera vez decididamente, asegurar que los mensajes de salud asociados a los alimentos respondan a la evidencia científica y que el consumidor pueda hoy confiar, mucho más que antes, en su veracidad. Pero hay que reconocer (probablemente no hubo otra forma mejor de abordarlo) que aún ha quedado bastante ambigüedad para muchos de los claims "funcionales" autorizados: los que se refieren al "manteni- 
miento normal" (o expresiones similares) de determinadas funciones generales, asociados a nutrientes esenciales. Así, se han aceptado claims muy generales (no específicos) de salud, pero sólo para vitaminas y minerales esenciales (que han sido los más "ganadores" en este proceso). Para estos nutrientes se siguen criterios diferentes de los aplicados a otros bioactivos (tal como prevé la normativa); en su evaluación se ha tenido en cuenta la existencia de mucha evidencia científica, largamente consolidada, con aportaciones mecanísticas muy sólidas en los casos en que no había estudios de intervención en humanos; pero lo cierto es que en muchos casos no hay constancia de que la dieta de la inmensa mayoría de europeos sea deficiente en la mayoría de estos nutrientes esenciales. Así, el avance ha sido muy importante, sobre todo en cuanto a garantizar la "veracidad" de los mensajes, pero no tanto en cuanto a facilitar suficientemente la comprensión de los mismos por el consumidor. Esto último requerirá de esfuerzos, principalmente desde otro ángulo, para conseguir una mayor formación del consumidor en estos temas de alimentación y nutrición, tan esenciales para su salud y bienestar. Principales retos de cara al futuro, y que son el centro de las consideraciones para la reevaluación del Reglamento de cara a su futura actualización, son la aplicación de criterios apropiados para la evaluación de los productos 'botánicos' (véase anteriormente), y la controversia y difícil solución para el establecimiento de los perfiles nutricionales.

El Reglamento prevé que sólo los alimentos con perfiles nutricionales "apropiados" puedan llevar declaraciones de salud. El concepto de perfil nutricional es, sin embargo, controvertido y de difícil traducción en la práctica (Palou, A., 2011; EFSA Panel on Dietetic Products Naan, 2008), como lo demuestra los muchos años de retraso que lleva su concreción, respecto de la fecha límite (19 de enero de 2009) que el Reglamento estableció para su aprobación (BIOCLAIMS, 2010). No ha sido posible concretarla. El Reglamento prevé prohibiciones de declaraciones nutricionales o de salud para aquellos alimentos que no respondan a un "perfil nutricional" mínimamente saludable. La opinión de la EFSA hace énfasis, de manera general, en unos pocos componentes: sobretodo grasa saturada y sal o sodio y también azúcar, mencionando otros parámetros como los relativos a la densidad energética que incluirían la composición en grasa total (y también los azúcares sencillos), todo ello con una aplicabilidad general a todo tipo de alimentos, pero sin descartar excepciones o derogaciones a grupos o tipos de alimentos, por ejemplo dependiendo de su carácter líquido o sólido, u otros aspectos de interés, incluyendo la dosis según los hábitos generales de consumo. La idea subyacente es que, dado que las declaraciones de salud pueden estimular el consumo de aquellos alimentos que las lleven, no parece razonable que tales alegaciones beneficien el consumo de alimentos con un perfil nutricional inadecuado. Sin embargo, hay muchos factores que inciden (EU, 2007; EFSA Panel on Dietetic Products Nutrition and Allergies, 2008) y creemos que este concepto de Perfil Nutricional está bastante abierto a una progresiva (pero lenta) evolución, cuyo ritmo vendrá dictado por criterios de aceptabilidad y aplicabilidad práctica en nuestra sociedad. La adopción de ta- 
les criterios es difícil, teniendo en cuenta lo previsto en el propio Reglamento sobre tener en cuenta toda la variabilidad de hábitos y tradiciones dietéticas en Europa, las posibilidades de innovación, el papel que determinados productos concretos tienen en la alimentación o en el contexto de una dieta global, etc. (EU, 2007; EFSA Panel on Dietetic Products Naan, 2008); es decir, son numerosos condicionantes, y van bastante más allá del campo puramente científico. Quizás el más importante a considerar a la hora de matizar posibles restricciones sea la dosis o las combinaciones, o incluso un mejor conocimiento de la relación entre riesgos y beneficios, en la medida en que un determinado alimento o componente incida sobre la dieta en su conjunto.

En cuanto a los nutrientes seleccionables para tener más en cuenta en un perfil nutricional, pueden incluirse tanto nutrientes que pueden y no deben ser consumidos en exceso (grasa, ácidos grasos saturados, ácidos grasos trans, sal/sodio y azúcar) como nutrientes cuya ingesta pueda ser inadecuada y sea recomendable potenciar su consumo en algunos grupos de población (fibra dietética, ácidos grasos n-3 y otros ácidos grasos insaturados, calcio, vitamina $D$, hierro, potasio y folato). En el futuro cabe esperar una consideración más amplia y no contemplamos a corto/medio plazo la implantación de los perfiles tal como han sido concebidos.

Por último, no se nos escapa la importancia de implicar a las nuevas tecnologías, la nutrigenómica en particular, a la hora de evaluar los aspectos de la alimentación relacionados con la salud, pues tanto los condicionantes genéticos como los epigenéticos definen respuestas particulares de las personas a los alimentos, y esta respuesta individual es la que afecta a cada persona, mientras que hoy aún prevalecen las estimaciones de los efectos de los alimentos y sus componentes, a nivel sólo de sus efectos considerados estadísticamente, para subpoblaciones amplias o para el conjunto de la población.

\section{REFERENCIAS}

BIOCLAIMS (2010). BIOmarkers of Robustness of Metabolic Homeostasis for Nutrigenomics-derived Health CLAIMS Made on Food" (BIOCLAMS, Grant agreement no. 244995).

EFSA PANEL ON DIETETIC PRODUCTS NAAN (2007). Opinion of the Panel on Dietetic Products, Nutrition and Allergies (NDA) related to scientific and technical guidance for the preparation and presentation of the application for authorisation of a health claim. The EFSA Journal.;530:1-44.

EFSA PANEL ON DIETETIC PRODUCTS NUTRITION AND ALLERGIES (2008). Opinion of the Panel on Dietetic Products, Nutrition and Allergies (NDA) related to the setting of nutrient profiles for foods bearing nutrition and health claims pursuant to article 4 of the regulation (ec) ${ }^{\circ}$ no $1924 / 2006$. The EFSA Journal;644:1-44

EFSA PANEL ON DIETETIC PRODUCTS NAAN (2016). EFSA NDA Panel (EFSA Panel on Dietetic Products, Nutrition and Allergies), 2016. General scientific guidance for stakeholders on health claim applications. The EFSA Journal;14(1):38.

EU (2007). Corrigendum to Regulation (EC) No 1924/2006 of the European Parliament and of the Council of $20 \mathrm{De}$ cember 2006 on nutrition and health 
claims made on foods. Official Journal of the European Union.;L12:3-18.

PALOU, A. (2011) Nuevas oportunidades. Alimentación: ¿para quién, cuándo y cómo? ¿Hacia una alimentación óptima y personalizada? In: Jordana, Polledo \& Palou, eds. Implicación Social de la Industria
Alimentaria. Barcelona: Ergon: pp. 72-78. PALOU, A.; PICO, C. \& BONET, M.L. (2004). Food safety and functional foods in the European Union: obesity as a paradigmatic example for novel food development. Nutrition Reviews;62(7 Pt 2):S169-181. 